This is the accepted version of the article:

Zhang F., Zhao T., Ruiz-Molina D., Liu Y., Roscini C., Leng J., Smoukov S.K.. Shape Memory Polyurethane Microcapsules with Active Deformation. ACS applied materials \& interfaces, (2020). 12. : 47059 - . 10.1021/acsami.0c14882.

Available at: https://dx.doi.org/10.1021/acsami.0c14882 


\title{
Shape Memory Polyurethane Microcapsules with Active Deformation
}

\author{
Fenghua Zhang ${ }^{a, b}$, Tianheng Zhao ${ }^{\text {b,c }}$, Daniel Ruiz-Molina ${ }^{d}$, Yanju Liu ${ }^{\mathrm{e}}$, Claudio \\ Roscini $^{\text {d,* }}$, Jinsong Leng ${ }^{a, *}$ and Stoyan K. Smoukov,f,* \\ ${ }^{a}$ Centre for Composite Materials and Structures, Harbin Institute of Technology (HIT), No. 2 YiKuang \\ Street, PO Box 3011, Harbin 150080, People`s Republic of China \\ ${ }^{b}$ Department of Materials Science and Metallurgy, University of Cambridge, 27 Charles Babbage Road, \\ Cambridge, CB3 0FS, UK \\ ${ }^{\mathrm{c}}$ Department of Chemistry, University of Cambridge, Lensfield Road, Cambridge, CB2 1EW, UK. \\ ${ }^{\mathrm{d}}$ Catalan Institute of Nanoscience and Nanotechnology (ICN2), CSIC and BIST, Campus UAB, Bellaterra, \\ 08193 Barcelona, Spain \\ ${ }^{\mathrm{e}}$ Department of Astronautical Science and Mechanics, Harbin Institute of Technology (HIT), No. 92 West \\ dazhi Street, PO Box 301, Harbin 150001, People`s Republic of China \\ ${ }^{\mathrm{f}}$ School of Engineering and Materials Science, Queen Mary University of London, Mile End Road, London, \\ E1 4NS, UK \\ *Author to whom any correspondence should be addressed. E-mail: s.smoukov@qmul.ac.uk, \\ lengjs@hit.edu.cn, claudio.roscini@icn2.cat
}

\section{ABSTRACT}

From smart self-tightening sutures and expandable stents to morphing airplane wings, shape memory structures are increasingly present in our daily life. The lack of methods for synthesizing intricate structures from them on the micron and sub-micron level, however is stopping the field from developing. In particular, the methods for synthesis of the shape memory polymers and structures at this scale and the effect of new geometries remain unexplored. Here we describe the synthesis of shape-memory polyurethane (PU) capsules accomplished by interfacial polymerization of emulsified droplets. The emulsified droplets contain the monomers for the hard segments, while the continuous aqueous phase contains the soft segments. A tri-functional chemical crosslinker for shape-memory PU synthesis was utilized to eliminate creep and improve recovery ratios of the final capsules. We observe an anomalous dependence of the recovery ratio with the amount of programmed strain compared to previous shape-memory polymers. We develop quantitative characterization methods and theory to show that when dealing with thin shell objects, alternative parameters to quantify recovery ratios are needed. We show that while achieving 94-99\% area recovery ratios, linear capsule recovery ratios can be as low as $70 \%$. This quantification method allows us to convert from observed linear aspect ratios in capsules to find out unrecovered area strain and stress. The hollow structure of 
the capsules grants high internal volume for some applications (e.g. drug delivery) which benefits from much higher loading of active ingredients than polymeric particles. The methods we developed for the capsule synthesis and programming could be easily scaled up for larger volume applications.

Keywords: Polyurethane microcapsules, shape memory polymers, interfacial polymerization, core-shell structures, shape-changing behaviors

\section{INTRODUCTION}

Shape memory polymers (SMPs) are a class of smart materials. ${ }^{1-3}$ Their existing surgical applications (self-tightening sutures) ${ }^{4}$ only hint at their far-reaching potential and emerging areas including robotics, ${ }^{5,6}$ aerospace, ${ }^{7}$ smart clothing, ${ }^{8}$ soft and flexible electronic device, ${ }^{9,10} 4 \mathrm{D}$ printing, ${ }^{11,12}$ and biomedical engineering ${ }^{13-15}$ (e.g. drug carrier, ${ }^{16}$ Orthoses, ${ }^{17}$ Occlusion Devices, ${ }^{18}$ stents, $\left.{ }^{19}\right)$. The materials exhibit programmed shape change in response to various external stimuli, including heat, water, solvents, electric/magnetic fields, microwave, ultrasound, light, pressure etc. ${ }^{20-23}$ Drawing on cross-discipline research from fields including mechanics, materials, chemistry, biomedicine, etc many macro-scale SMPs and their composites have been developed. In addition to programmable shapes, shape-memory polymers have been incorporated in actuators, yielding programmable movement. ${ }^{24}$ Recently we have developed a combinatorial strategy to incorporate multiple functions in polymer networks by simultaneous or successive interpenetration. ${ }^{25}$ The trend towards miniaturization opens opportunities for SMP nano/micro-sized actuators for biomedical applications, e.g. the defined positioning of medical devices in brain surgery or reduced reagent consumption in bioanalytics. Microscopic devices with shape changing structures may provide new concepts of drug targeting and release/delivery using shape dependent lock-and-key biorecognition. $^{26}$ Though a large number of macro-scale structures and morphologies have been designed from many different polymers showing the shape memory effect, when scaling SMPs devices to the micrometer level, it is a challenge to maintain the shape memory function. Emulsified droplets may provide a promising bottom-up 
approach to fabricate such functional micro- and nano-particles, ${ }^{27}$ a strategy also compatible with polymerization. $^{28}$

Current research on shape memory effects is focusing on bulk film, fiber or foam polymer materials. ${ }^{29-31}$ For the microstructure, mainly micropatterning the surface of an SMP film was investigated. ${ }^{32,33}$ Micro-/nanoparticles with shape memory properties had limited prior studies. Non-spherical polymer colloid nanoparticles with shape memory effect have been reported in 2005. ${ }^{34}$ Notably, Lendlein's group developed shape memory micro-/nanoparticles used for drug delivery. ${ }^{35}$ These previous studies demonstrated a promising application of such materials in programmable pharmaceuticals, but a major challenge is the limited drug loading, which hollow capsules can help to overcome.

Therefore we designed and synthesized a class of polyurethane (PU) core/shell microcapsules that demonstrate the shape memory effect. As far as we know this phenomenon has not been described before with core/shell capsules. The merits of these microcapsules include easy fabrication, tunable morphologies, large deformation, and excellent shape memory properties. The shape memory polyurethane (SMPU) microcapsules were fabricated via initial emulsification of reagents separated in oil/water phases, followed by interfacial polymerization process. The effect of the crosslinking of the polymer shell on the programmable behavior of shapes was investigated. Importantly, the change from film to sphere-like geometry has resulted in the need for alternative methods to characterize the capsules' shape memory performance to be compared more consistently.

\section{EXPERIMENTAL SECTION}

Fabrication of core/shell polyurethane microcapsules. SMPU microcapsules were fabricated in different conditions (Table 1). $0.252 \mathrm{~g}$ of the aliphatic triisocyanate Desmodur N 3300 (DN 3300, molecular weight is 504, Bayer) were added into $5 \mathrm{ml}$ of dioctyl terephthalate (DOT, Sigma-Aldrich) oil. $1.15 \mathrm{~g}$ of polyethyleneglycol (PEG, molecular weight is $4600 \mathrm{~g} / \mathrm{mol}$ ) were dissolved into $1 \mathrm{wt} \%$ polyvinyl alcohol (PVA, 86\%-89\% hydrolyzed, medium molecular weight, Sigma-Aldrich) deionized (DI) water solution (water phase). The two solutions were mixed together and emulsified at different rates (i.e. 5 000, 10 000, and $15000 \mathrm{rpm}$ ), for $15 \mathrm{~min}$ at room temperature, using IKA 
Ultra-Turrax homogenizer. The obtained emulsion was transferred into a three-neck flask, which was heated by an oil bath to keep the temperature at $65^{\circ} \mathrm{C}$ for 30 min under nitrogen atmosphere. Finally, $0.059 \mathrm{~g}$ of the chain extender 1,4-butanediol (BDO) (Sigma-Aldrich) were added into the flask and the temperature was increased to $80{ }^{\circ} \mathrm{C}$ for $4 \mathrm{~h}$. SMPU microcapsules were also obtained by replacing the DN 3300 with equal number of moles of polyisocyanate Desmodur N 100 (DN 100). SMPU microcapsules were washed by DI water for 5 times and collected by centrifuge to separate the capsules from the water phase (12 $000 \mathrm{rpm}$ for $30 \mathrm{~min}$ ).

Table 1 SMPU capsules fabricated in different conditions.

\begin{tabular}{|c|c|c|c|c|c|}
\hline Sample & $\begin{array}{l}\text { Hard segment:Soft } \\
\text { segment Mole Ratio }\end{array}$ & $\begin{array}{l}\text { Oil Phase } \\
\qquad 5 \mathrm{~mL}\end{array}$ & $\begin{array}{c}\text { Water Phase } \\
40 \mathrm{~mL} \\
1 \% \text { PVA }\end{array}$ & $\begin{array}{l}\text { Speed } \\
(\mathrm{rpm})\end{array}$ & $\begin{array}{l}\text { Time } \\
(\min )\end{array}$ \\
\hline SMPU1 & $2: 1$ & DN 3300 & PEG 4600 & 5000 & 15 \\
\hline SMPU2 & $2: 1$ & DN 3300 & PEG 4600 & 10000 & 15 \\
\hline SMPU3 & $2: 1$ & DN 3300 & PEG 4600 & 15000 & 15 \\
\hline SMPU4 & $2: 1$ & DN100 & PEG 4600 & 15000 & 15 \\
\hline
\end{tabular}

Shape memory behavior of microcapsules. The resulting SMPU microcapsules were added into $5 \mathrm{ml} 10 \mathrm{wt} \%$ PVA solution (PVA 4-88, Sigma Aldrich), which was poured onto a 8.5 diameter Petri dish. After evaporating the water, a solid PVA film was obtained and was stretched to different degrees at $80{ }^{\circ} \mathrm{C}$ and $10{ }^{\circ} \mathrm{C} / \mathrm{min}$ (using DMA in tensile model, TA). The PVA of the programmed film was dissolved in DI water to obtain a suspension of microcapsules with ellipsoidal shape (the temporary shape). When reheating the programmed microcapsules suspension or powder above the shell polymer transition temperature, they returned from the ellipsoidal (temporary) shape to the spherical (permanent) shape.

Characterization. The morphology and structure of microcapsules was characterized using scanning electron microscopy (SEM, FEI Nova Nano) with sputtering Pt for 1 min. The mean diameters of microcapsules in different conditions were calculated by Image $\mathrm{J}$. 
The shape recovery process was monitored by putting the stretched SMPU microcapsules on a heat stage with circulating water with temperature above the transition temperature of the microcapsules shell polymer, observing and recording the shape recovery process with fluorescence optical microscopy (Zeiss Axio Observer Z1m inverted optical microscope, with an $\mathrm{Hg}$ lamp excitation source) at different magnifications. The videos and the images of the fluorescent microscapsules were obtained with the AxioCamHRc digital camera. Differential scanning calorimetry (DSC, TA) was carried out to test the thermal properties of the capsules from $-80{ }^{\circ} \mathrm{C}$ to $100{ }^{\circ} \mathrm{C}$ at a heating and cooling rate of $10{ }^{\circ} \mathrm{C} / \mathrm{min}$ in a nitrogen atmosphere.

\section{RESULTS AND DISCUSSION}

In this work a series of multiblock copolymer SMPU capsules with urethane linkages (NHCOO-) were synthesized by using one of the two aliphatic triisocyanates Desmodur N 100 (DN 100) and Desmodur N 3300 (DN 3300). The chemical structure of the different monomers and synthesis procedure and chemical structure of SMPU are shown in Figure S1 and Figures S2 (Supporting information). Dioctyl terephthalate (DOT), a high boiling point oil, was used as core materials in the microcapsules, which guarantees the capsules thermal stability during the heating required for their stretching and shape recovery. Figure 1 shows the schematic representation of the three-step preparation of the core/shell SMPU microcapsules ( DN 3300-based SMPU capsules are showed as example): i) the oil-in-water emulsification was carried out by homogenizing the lipophilic DN 3300/DOT(or DN 100/DOT) solution with the aqueous phase containing the stabilizer and the hydrophilic monomer, yielding microsized oil droplets in water; $i$ ) interfacial polymerization where the lipophilic monomer (DN 3300 or DN 100) reacts with the hydrophilic monomer (PEG) in the oil/water interface forming the initial shell material around the microdroplets; and iii) a second crosslinking of the polymer shell upon addition of the chain extender BDO. A lipophilic fluorescent dye, dissolved in the initial oil phase, was used to allow an easier detection of the shape change of the small capsules. In SMPU capsule systems, isocyanates act as the hard segment responsible for the permanent shape. The semicrystalline polyethylene glycol (PEG) serves as switchable phase that controls the temporary shape. The transition temperature is determined by the 
melting temperature of the PEG. The chemical structure of isocyanates plays an important role in the network and shape recovery behavior.

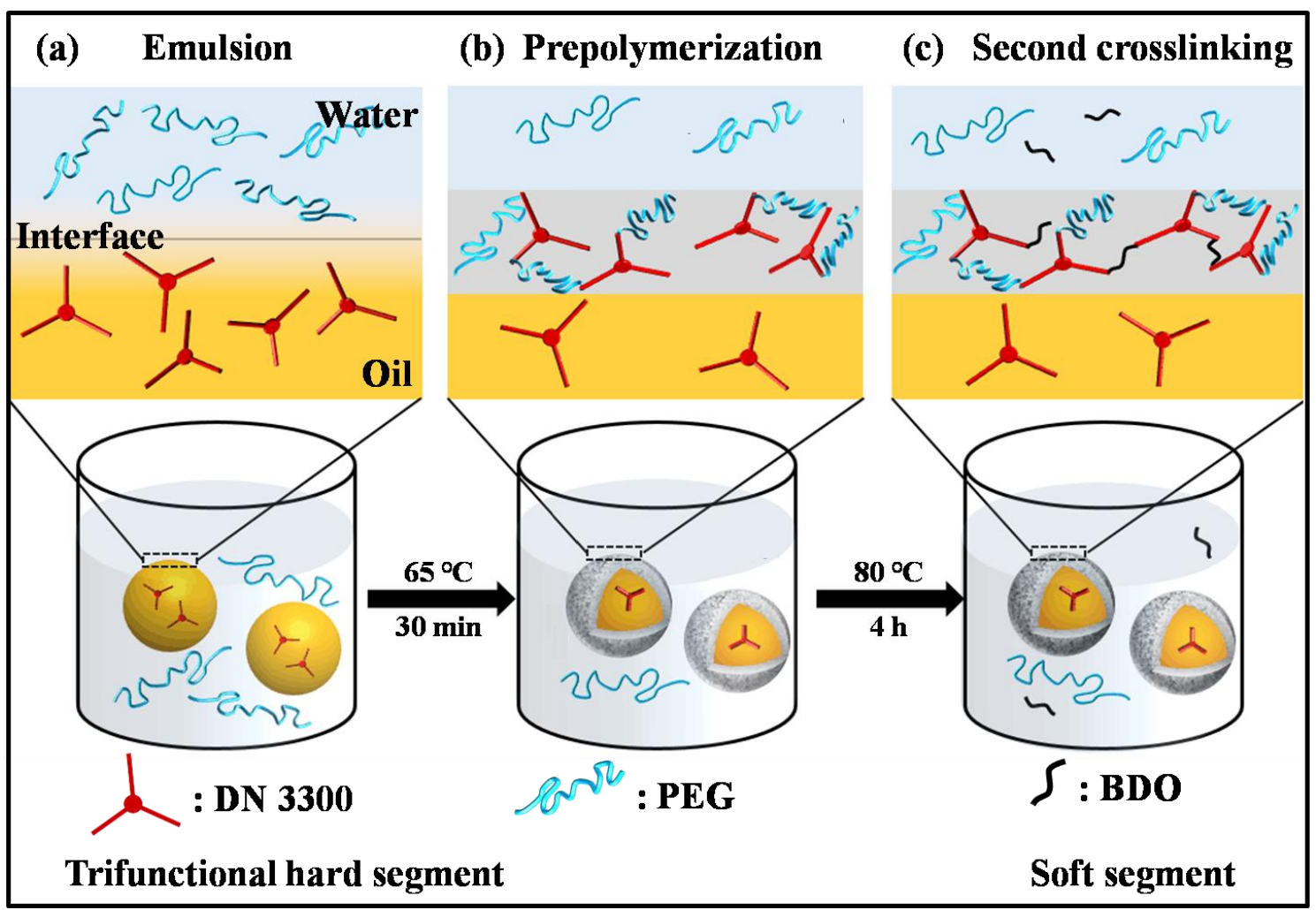

Figure 1. Schematic illustration of the fabrication of SMPU capsules. a) water phase with PEG and PVA and the oil phase with DN 3300 and DOT were homogenized using an IKA Ultra-Turrax for 15 min to make uniform oil microdroplets; b) the emulsion was stirred and heated at $65{ }^{\circ} \mathrm{C}$ for $30 \mathrm{~min}$, and the $-\mathrm{CNO}$ groups of DN3300 were linked to the chain ends of the PEG at the oil-water interface; c) BDO was added in the water phase and the reaction temperature changed to $80{ }^{\circ} \mathrm{C}$ for $4 \mathrm{~h}$ to allow a second crosslinking reaction between DN3300 and BDO and obtain the final core/shell microcapsules.

Under various reaction conditions (Table 1), we successfully obtained several SMPU capsules with different diameters. Figures 2a-2c show SEM images of SMPU microcapsules exhibiting smooth surfaces. The mean diameter of these capsules was controlled by the stirring speed in the emulsification step. Figure 2d shows the diameter distribution indicating that the mean diameter decreases from $9 \mu \mathrm{m}$ to $1.5 \mu \mathrm{m}$ with stirring speed increase from $5000 \mathrm{rpm}$ to $15000 \mathrm{rpm}$. The capsule size can be further controlled by factors such as stirring time for emulsification, and surfactant used (Table 1). From mechanically crushed or uncompleted capsules that partially or completely 
released their core materials, it was possible to corroborate their core-shell structure and estimate the shell thickness, as previously showed, ${ }^{36-38}$ which ranged from $300 \mathrm{~nm}$ to 950 $\mathrm{nm}$, depending on the capsules size (Figure 2e and S3). For the large capsules, attempts of measure the shell thickness through TEM failed due to the high electron density and large electron pathway through the capsule volume, which make the shell and the core materials impossible to differentiate. For the smaller capsules, the thickness can be observed clearly (Figure S4). As shown in Figure 2f, the melting temperature $\left(T_{m}\right)$ of DN 3300 based SMPU microcapsules shell polymer is around $55^{\circ} \mathrm{C}$ and $\Delta H_{\mathrm{m}}$ is $11.60 \mathrm{~J} / \mathrm{g}$. The $T_{m}$ is low enough to allow stretching of the microcapsules without requiring very high temperature, which could cause degradation of encapsulated materials. The capsule size did not affect the $\mathrm{T}_{\mathrm{m}}$ of the capsules shell.

Furthermore, in order to evaluate the effect of the monomer structure on the thermal properties of the microcapsules and their ability to recover their shape, we fabricate the DN 100 based SMPU microcapsules with different diameters (Figure S5, Supporting Information).

It must be noticed that in all cases the isocyanate functionality might undergo competing reactions with the water and PVA molecules of the aqueous phase. The reaction with $\mathrm{H}_{2} \mathrm{O}$ molecules yields amines, which can then react with isocyanate groups forming polyurea. However, due to the reduced water reactivity, the amount of polyurea formed in the shell polymer is negligible. In the same way, the reactivity of the isocyanate functionality with the PVA stabilizer is negligible, possibly due to the more hindered hydroxyl group, compared to PEG and BDO. Therefore, most of the polymer structure is made by crosslinked polyurethane formed from the reaction of the isocyanates (DN3300 or DN 100) and the alcohols (PEG and BDO). 

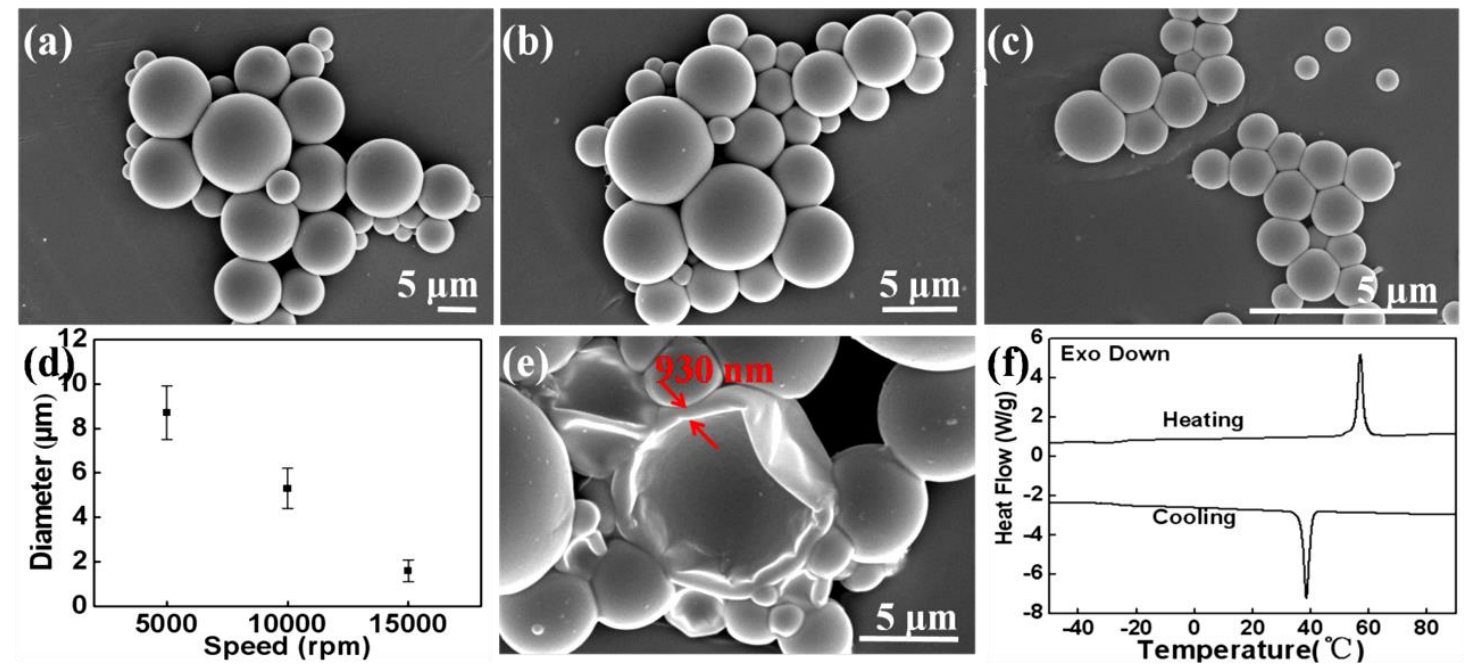

Figure 2. Characterization of microcapsule produced at different stirring conditions. a)-c) SEM images of SMPU microcapsules generated from $5000 \mathrm{rpm}, 10000 \mathrm{rpm}$, and $15000 \mathrm{rpm}$, respectively. d) Average diameter of SMPU microcapsules produced at different stirring speeds, showing a negative proportional relationship between speed and average size. e) Representative SEM image of the PU microcapsule, showing the core-shell structure with partially empty void and a wall thickness of $930 \mathrm{~nm}$ for a large microcapsule. f) DSC thermogram of SMPU capsules, including cooling and second heating curves.

The scheme illustrating the shape programming and shape recovery processes of the microcapsules is shown in Figure 3a. The microcapsules were stretched by embedding them in a polymer film, which is elongated while heated above its glass transition temperature $\left(\mathrm{T}_{\mathrm{g}}\right)$. Polyvinyl alcohol (PVA) was chosen as the polymer matrix because: $\mathrm{i}$ ) it is a thermaoplastic film-forming polymer which can trap microcapsules using the casting method; ${ }^{37-39}$ ii) it can be stretched when heated above its $T_{g}$; iii) it has a $T_{g}$ around $70{ }^{\circ} \mathrm{C}$ (Figure S6), i.e. it can be heated at higher temperature than the $\mathrm{T}_{\mathrm{m}}$ of PU, allowing to reach the melting point of the PU shell polymer, and therefore the thermallyinduced capsules deformation upon film stretching; iv) it is water-soluble, which means it allows to recover and characterize the deformed microcapsules upon film dissolution. The microcapsules were dispersed into PVA solution, which was then cast onto a Petri dish. A solid film with capsules embedded in was obtained when the water evaporated. The formed film was heated at $80{ }^{\circ} \mathrm{C}$, to assure a temperature above both the $\mathrm{T}_{\mathrm{g}}$ of PVA 40 and the $\mathrm{T}_{\mathrm{m}}$ of PU transition temperatures. Capsules with temporary fixed ellipsoidal shape were obtained after dissolving the stretched PVA film in the water at room temperature. The stretched ellipsoidal capsules and the thermally-induced shape recovery process were monitored with fluorescence microscopy. 

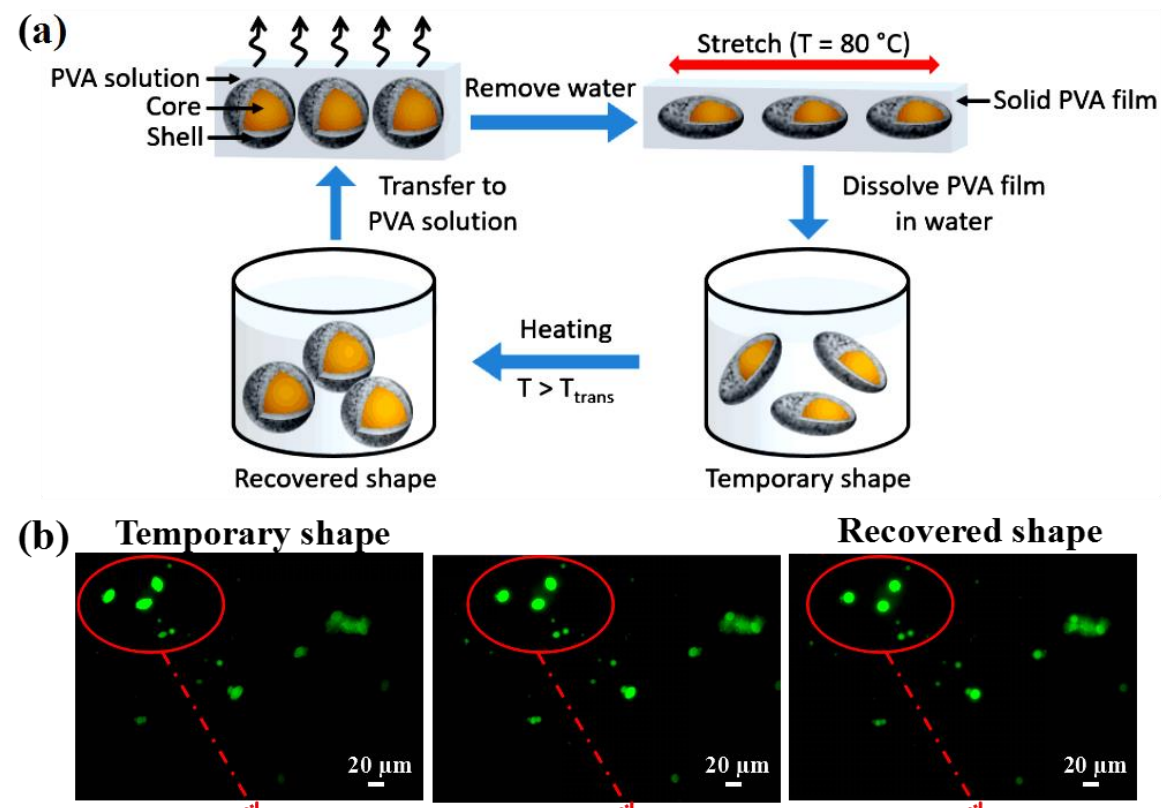

Recovered shape
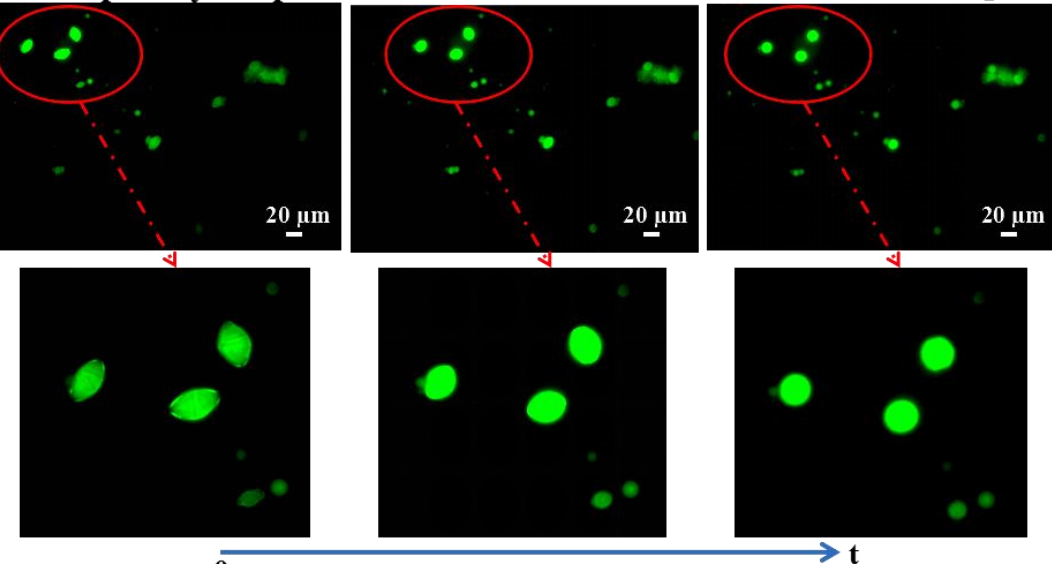

0

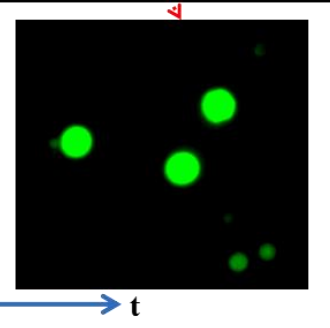

Figure 3. Shape memory behaviors of the synthesized microcapsules. a) Schematic illustration of the programming and recovery process of SMPU microcapsules. The microcapsules were first embedded in a PVA film to thermally induce their stretching. The isolated programmed microcapsules are obtained after dissolving the PVA in water. At $\mathrm{T}>\mathrm{T}_{\mathrm{m}}$, the microcapsules recover their original shape. The whole process can then be repeated. b) Fluorescent images of the programmed and shape recovery behavior of SMPU capsules. The aspect ratio changed from 1.66 for the temporary shape to 1.06 for the recovered shape. The shape recovery happened at $60{ }^{\circ} \mathrm{C}$ and took about $30 \mathrm{~s}$ to complete.

The ellipsoidal (temporary) shapes of SMPU microcapsules can be fixed according to the above-mentioned method. We also demonstrated the shape recovery behavior. Taking the SMPU microcapsules stretched to $80 \%$ for example, Figure $3 \mathbf{b}$ shows the shape recovery process of DN 3300 based SMPU microcapsules. The ellipsoidal capsules were placed in a water bath. Heating the sample to $60{ }^{\circ} \mathrm{C}$, the ellipsoidal shape capsules recover the spherical shape after several seconds with a shape recovery ratio of $88.5 \%$. The whole process was recorded in a fluorescence microscope (See Movie S1, Supporting information). DN100-based SMPU capsules also exhibit excellent shape recovery properties (Figure S7, Movie S2, Supporting information). 
For a quantitative description of programming and recovery of the SME capsules, we measured the aspect ratio (AR) of the longest axis $l$ to the shortest axis $d$. Prolate spheroids (temporary shape) were obtained by stretching phantoms to different ratios. $A R_{\text {programmed }}$ is the aspect ratio for deformed capsules, $A R_{\text {recovered }}$ is the aspect ratio for recovered capsules. For the original spherical shape, the $A R_{\text {original }}=1$. 

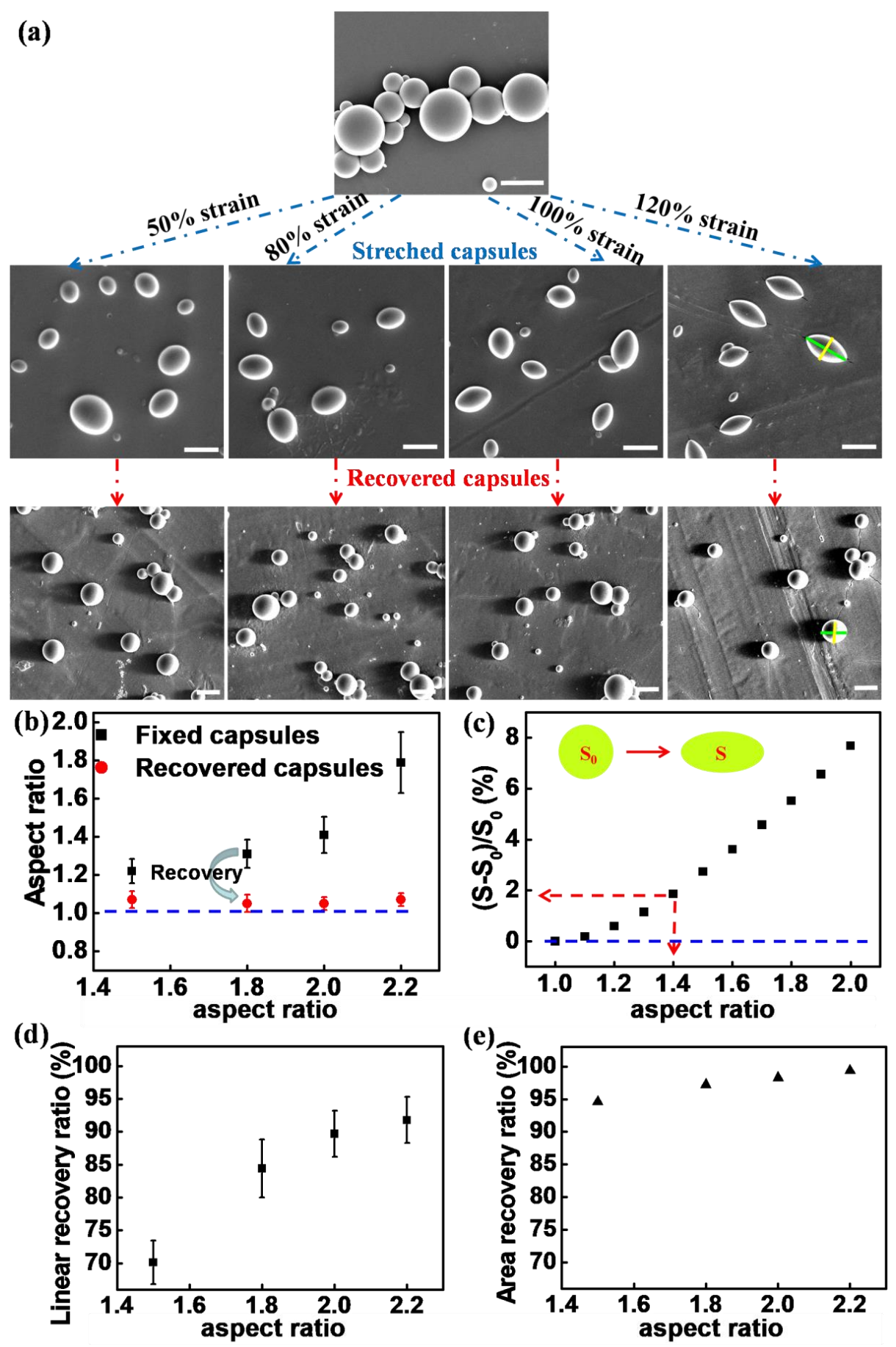

Figure 4. Detailed analysis of recovery status for microcapsules programmed to different stains. a) SEM images of SMPU microcapsules with different stretching ratios $(50 \%, 80 \%, 100 \%$ and 120\%) and shape recovery after heating. The images of the stretched and shape recovered capsules are taken from aliquots of the same suspension, before and after heating. All scale bars are $10 \mu \mathrm{m}$. b) The aspect ratio of deformed and recovered microcapsules at different stretching ratios. All the samples successfully regained near spherical shape with the aspect ratio close to 1 . c) Continuously increasing surface area ratio between the stretched ellipsoid and the recovered shape. d) Trend of liner recovery ratio for stretched capsules at different stretched ratio. Hypothesis: Sphere volume=Ellipsoid volume; radius is 1, units have no effect on this trend. e) Surface area increase percent of stretched capsules at different stretching ratios based on the constant volume hypothesis. 
In order to investigate quantitatively the shape memory effect of SMPU microcapsules, we stretched the PVA film embedding the microcapsules to different strains (increasing the film size 50\%, 80\%, 100\%, 120\%, 150\% and $200 \%$ respect to the original length). The optical microscope images of DN 3 300-based SMPU capsules (with fluorescent dye) in PVA matrix (Figure S8). Figure 4a shows SEM images of SMPU microcapsules subjected to different degrees of elongation $(50 \%, 80 \%, 100 \%$, and $120 \%$ ), yielding ellipsoidal microcapsules, and the respective recovered spherical shape after thermal treatment. When stretching the capsules to $150 \%$, some microcapsules begin to break (Figure S9a, Supporting information). All microcapsules broke after stretching to $200 \%$ (Figure S9b, Supporting information). The shape fixity ratios are higher than $80 \%$ (as shown in Figure S10). When reheating the ellipsoidal capsules (no break) above the $\mathrm{T}_{\mathrm{m}}$ of SMPU $\left(60{ }^{\circ} \mathrm{C}\right)$, the pre-deformed capsules recovered their spherical shapes. The change of aspect ratios after the shape recovery process is shown in Figure 4b. Regardless of the programmed aspect ratio, SMPU microcapsules exhibit good shape memory properties and effective return to spherical shape, with recovered aspect ratios near 1 . The surface area of deformed ellipsoids were calculated based on the measured aspect ratios (Figure 4c), and the detailed calculation are shown in the supporting information. For drug delivery applications, the surface area strongly affects the diffusion rate of the internal content. With such a large surface area change, the capsule has the potential to modulate the drug release rate. The linear shape recovery ratios at different stretch ratios are shown in Figure 4d. Notably, this trend differs greatly from those previously reported for film geometries. Therefore, it is necessary to establish a new method to properly evaluate and compare the shape recovery efficiency for microcapsules and nanocapsules. We propose to compare the recovered surface area and the theoretical surface area of the original sphere, and such method yields results that are more closely comparable to those reported for thin films. The surface area recovery ratios, as the linear recovery ratios, also increase monotonically with increase in programmed aspect ratio, but their values are much higher, close to $100 \%$, Figure 4 e. These are values expected for crosslinked polyurethane films and put in perspective the observed linear recovery ratios. 


\section{CONCLUSIONS}

In summary, we describe a general method for synthesizing polyurethane shape-memory microcapsules. Based on emulsification and interfacial polymerization, it is a scalable method to obtain microcapsules with potential applications in drug delivery. The synthesis of the polyurethane microcapsules is based on aliphatic triisocyanates (both DN 3300 and DN 100), which grants excellent shape memory effects. We show that the molecular structures of the chemical monomers play an import role in controlling the crosslinking density, which determined the shape recovery properties of SMPs and microstructures made from them. The shell geometry of the polymer in these microcapsules leads to non-linear responses of these structures compared to shapememory polymer films. We have therefore proposed novel methods to relate the change in apparent aspect ratio of the capsules to surface area changes instead, which allows a closer comparison of shape memory behavior between the microcapsule to the traditional polymer films.

\section{ASSOCIATED CONTENT}

\section{Supporting Information.}

The Supporting Information is available free of charge via the Internet at http://pubs.acs.org.The following files are available. Details of materials used; characterization; supplementary figures and tables (PDF). Movies of the shape recovery process (Movie 1 and Movie 2)

\section{AUTHOR INFORMATION}

\section{Corresponding Author}

*E-mail: $\underline{\text { s.smoukov@qmul.ac.uk, lengjs@ hit.edu.cn, claudio.roscini@icn2.cat }}$

\section{Author Contributions}

The manuscript was written through contributions of all authors. All authors have given approval to the final version of the manuscript. 


\section{Notes}

The authors declare no competing financial interest.

\section{ACKNOWLEDGMENTS}

This work acknowledges support by the National Natural Science Foundation of China (Grant Nos.11632005, 11672086, 11802075), by the European Research Council (EMATTER \#280078), and by the UK EPSRC (Fellowship No. EP/R028915/1). Fenghua Zhang would like to thank Chinese Scholarship Council (CSC) for funding her research work at the University of Cambridge. This work was also supported by grants from the Spanish Research Agency funds (AEI, grant no. RTI2018-098027-B-C21) by the European Regional Development Fund (ERDF) and BIST (BIST Ignite Project). The ICN2 is funded by the CERCA programme/Generalitat de Catalunya. The ICN2 is supported by the Severo Ochoa Centres of Excellent programme, funded by the Spanish Research Agency (AEI, grant no. SEV-2017-0706).

\section{REFERENCES}

(1) Leng, J. S.; Lan, X.; Liu, Y. J.; Du, S. Y. Shape-Memory Polymers and Their Composites: Stimulus Methods and Applications. Prog. in Mater. Sci. 2011, 56, 1077-1135.

(2) Liu, C.; Qin, H.; Mather, P. T. Review of Progress in Shape-Memory Polymers. J. Mater. Chem. 2007, $17,1543-1558$.

(3) Meng, H.; Li, G. Q. A Review of Stimuli-Responsive Shape Memory Polymer Composites. Polymer, 2013, 54, 2199-2221.

(4) Lendlein, A.; Langer, R. Biodegradable, Elastic Shape-Memory Polymers for Potential Biomedical Applications. Science 2002, 296, 1673-1676.

(5) Mousavi, S.; Howard, D.; Zhang, F. H.; Leng, J. S.; Wang, C. H. Direct 3D Printing of Highly Anisotropic, Flexible, Constriction Resistive Sensors for Multidirectional Proprioception in Soft Robots. ACS Appl. Mater. Inter. 2020, 12, 15631-15643

(6) Jin, B.; Song, H.; Jiang, R.; Song, J.; Zhao, Q.; Xie, T. Programming a Crystalline Shape Memory Polymer Network with Thermo- and Photo-Reversible Bonds toward a Single-Component Soft Robot. Sci. $A d v .2018,4$, eaao3865.

(7) Liu, Y. J.; Du, H. Y.; Liu, L. W.; Leng, J. S. Shape Memory Polymers and Their Composites in Aerospace Applications: A Review. Smart Mater. Struct. 2014, 23, 023001.

(8) Hu, J. L.; Meng, H.; Li, G. Q.; Ibekwe, S. I. A review of Stimuli-Responsive Polymers for Smart 
Textile Applications. Smart Mater. Struct. 2012, 21, 053001.

(9) Gao, H.; Li, J. R.; Zhang, F. H.; Liu, Y. J.; Leng, J. S. The Research Status and Challenges of Shape Memory Polymers in Flexible Electronics and Structural Devices. Mater. Horizons 2019, 6, 931-944.

(10) Avendano-Bolivar, A.; Ware, T.; Arreaga-Salas, D.; Simon, D.; Voit, W. Mechanical Cycling Stability of Organic Thin Film Transistors on Shape Memory Polymers. Adv. Mater. 2013, 25, 3095-3099.

(11) Zhang, Y.; Huang, L.; Song, H.; Ni, C.; Wu, J.; Zhao, Q.; Xie, T. 4D Printing of a Digital Shape Memory Polymer with Tunable High Performance. ACS Appl. Mater. Inter. 2019, 11, 32408-32413.

(12) Kuang, X.; Roach, D. J.; Wu, J. T.; Hamel, C. M.; Ding, Z.; Wang, T. J.; Dunn, M. L.; Qi, H. J. Advances in 4D Printing: Materials and Applications. Adv. Funct. Mater. 2019, 29, 1805290.

(13) Yakacki, C. M.; Gall, K. Shape-Memory Polymers for Biomedical Applications. Adv. Polym. Sci. 2010, 226, 147-175.

(14) Small, W.; Singhal, P.; Wilson, T. S.; Maitland, D. J. Biomedical Applications of Thermally Activated Shape Memory Polymers. J. Mater. Chem. 2010, 20, 3356-3366.

(15) Zhao, W.; Liu, L. W.; Zhang, F. H.; Leng, J. S.; Liu, Y. J. Shape Memory Polymers and Their Composites in Biomedical Applications. Mater. Sci. Eng. C 2019, 97, 864-883.

(16) Hribar, K. C.; Metter, R. B.; Ifkovits, J. L.; Troxler, T.; Burdick, J. A. Wischke, C.; Neffe, A. T.; Steuer, S.; Lendlein, A. Evaluation of a Degradable Shape-Memory Polymer Network as Matrix for Controlled Drug Release. J. Control. Release 2009, 138, 243-250.

(17) Chen, J.; Hu, J.; Leung, A. K. L.; Chen, C.; Zhang, J.; Zhang, Y.; Zhu, Y.; Han, J.; Shape Memory Ankle-Foot Orthoses. ACS Appl. Mater. Inter. 2018, 10, 32935-32941.

(18) Lin, C.; Lv, J., Li, Y.; Zhang, F. H.; Li, J.; Liu, Y. J.; Liu, L. W.; Leng, J. S. 4D-Printed Biodegradable and Remotely Controllable Shape Memory Occlusion Devices. Adv. Funct. Mater. 2019, 29, 1906569.

(19) Zhang, F. H.; Wang, L. L.; Zheng, Z. C.; Liu, Y. J.; Leng, J. S. Magnetic Programming of 4D Printed Shape Memory Composite Structures. Compos. Part A 2019, 125, 105571.

(20) Lendlein, A.; Jiang, H.; Junger, O.; Langer, R. Light-Induced Shape-Memory Polymers. Nature 2005, 434, 879-882.

(21) Li, W. B.; Liu, Y. J.; Leng, J. S. Selectively Actuated Multi-Shape Memory Effect of a Polymer Multicomposite. J. Mater. Chem. A 2015, 3, 24532-24539.

(22) Chen, H. M.; Li, Y.; Liu, Y.; Gong, T.; Wang, L.; Zhou, S. B. Highly Ph- Sensitive Polyurethane Exhibiting Shape Memory and Drug Release. Polym. Chem. 2014, 5, 5168-5174.

(23) Zhang, F. H.; Zhang, Z. C.; Luo, C. J.; Lin, I. T.; Liu, Y. J.; Leng, J. S.; Smoukov, S. K. Remote, Fast Actuation of Programmable Multiple Shape Memory Composites by Magnetic Fields. J. Mater. Chem. C 2015, 3, 11290-11293.

(24) Khaldi; A.; Elliott, J. A.; Smoukov, S. K. Electro-Mechanical Actuator with Muscle Memory. J. Mater. Chem. C 2014, 2, 8029-8034.

(25) Khaldi, A.; Plesse, C.; Vidal, F.; Smoukov, S. K. Smarter Actuator Design with Complementary and Synergetic Functions. Adv. Mater. 2015, 27, 4418-4422. 
(26) Champion, J. A.; Mitragotri, S. Role of Target Geometry in Phagocytosis. Proc. Natl. Acad. Sci. USA 2006, 103, 4930-4934.

(27) Denkov, N.; Tcholakova, S.; Lesov, I.; Cholakova, D.; Smoukov, S. K. Self-Shaping of Droplets via Formation of Intermediate Rotator Phases upon Cooling. Nature 2015, 528, 392-395.

(28) Lesov, I.; Valkova, Z.; Vassileva, E.; Georgiev, G. S.; Ruseva, K.; Simeonov, M.; Tcholakova, S.; Denkov, N. D.; Smoukov, S. K. Bottom-up Synthesis of Polymeric Micro- and Nanoparticles with Regular Anisotropic Shapes. Macromolecules 2018, 51, 7456-7462.

(29) Xie, T. Tunable Polymer Multi-Shape Memory Effect. Nature, 2010, 464, 267-270.

(30) Zhang, F. H.; Xia, Y. L.; Wang, L. L.; Liu, L. W..; Liu, Y. J.; Leng, J. S. Conductive Shape Memory Microfiber Membranes with Core-Shell Structures and Electroactive Performance. ACS Appl. Mater. Inter. 2018, 10, 35526-35532.

(31) Zhang, F. H.; Zhou, T. Y.; Liu, Y. J.; Leng, J. S. Microwave Synthesis and Actuation of Shape Memory Polycaprolactone Foams with High Speed, Sci. Rep. 2015, 5, 11152.

(32) Schauer, S.; Baumberg, J. J.; Holscher, H.; Smoukov, S. K. Tuning of Structural Colors Like a Chameleon Enabled by Shape- Memory Polymers. Macromol. Rapid Comm. 2018, 39, 1800518

(33) Xie, Y.; Meng, Y.; Wang, W.; Zhang, E.; Leng, J. S.; Pei, Q. B. Bistable and Reconfigurable Photonic Crystals-Electroactive Shape Memory Polymer Nanocomposite for Ink-Free Rewritable Paper. Adv. Funct. Mater. 2018, 28, 1802430.

(34) Yang, Z.; Huck, W. T.; Clarke, S. M.; Tajbakhsh, A. R.; Terentjev, E. M. Shape-Memory Nanoparticles from Inherently Non-spherical Polymer Colloids. Nat. Mater. 2005, 4, 486-490.

(35) Wischke, C.; Schossig, M.; Lendlein, A. Shape-Memory Effect of Micro-/Nanoparticles from Thermoplastic Multiblock Copolymers. Small 2014, 10, 83-87.

(36) Vazquez-Mera, N.; Roscini, C.; Hernando, J.; Ruiz-Molina, D. Liquid-Filled Capsules as Fast Responsive Photochromic Materials. Adv. Optical Mater. 2013, 1, 631-636.

(37) Julia-Lopez, A.; Hernando, J.; Ruiz-Molina, D.; Gonzalez-Monje P.; Sedo J.; Roscini, C. Temperature-Controlled Switchable Photochromism in Solid Materials. Angew. Chem. Int. Ed. 2016, 55, 15044-15048.

(38) Vazquez-Mera, N.; Otaegui, J. R.; Sanchez, R. S.; Prats, G.; Guirdao, G.; Ruiz-Molina, D.; Roscini, C; Hernando, J. Color-Tunable White-Light-Emitting Mateirals Based on Liquid-Filled Capsules and Thermally Responsive Dyes. ACS Appl. Mater. Interfaces 2019, 11, 17751-17758.

(39) Julia-Lopez, A.; Ruiz-Molina, D.; Hernando, J.; Roscini, C. Solid Materials with Tunable Reverse Photochromism. ACS Appl. Mater. Interfaces 2019, 11, 11884-11892.

(40) Torres-Pierna, H.; Ruiz-Molina, D.; Roscini, C. Highly Transparent Photochromic Films with a Tunable and Fast Solutio-like Response. Mater. Horiz. 2020, DOI 10.1039/d0mh01073a 